\title{
Detour Hosoya Polynomials of Some Compound Graphs
}

Herish O. Abdullah

herish_omer@yahoo.com
Gashaw A. Muhammed-Saleh

gashaw.mohammed@su.edu.krd

College of Science

University of Salahaddin

Received on:23/11/2009

Accepted on:11/4/2010

\section{ABSTRACT}

In this paper we will introduce a new graph distance based polynomial; Detour Hosoya polynomials of graphs $\boldsymbol{H}^{*}(\boldsymbol{G} ; \boldsymbol{x})$. The Detour Hosoya polynomials $\boldsymbol{H}^{*}(\boldsymbol{G} ; \boldsymbol{x})$ for some special graphs such as paths and cycles are obtained. Moreover the Detour Hosoya polynomials $\boldsymbol{H}^{*}\left(\boldsymbol{G}_{\mathbf{1}} \bullet \boldsymbol{G}_{\mathbf{2}} ; \boldsymbol{x}\right)$, $\boldsymbol{H}^{*}\left(\boldsymbol{G}_{\mathbf{1}}: \boldsymbol{G}_{2} ; \boldsymbol{x}\right)$ and $\boldsymbol{H}^{*}\left(\boldsymbol{G}_{\mathbf{1}} \odot \boldsymbol{G}_{2} ; \boldsymbol{x}\right)$ are obtained.

Keywords: Detour distance, compound graphs, Hosoya polynomials.

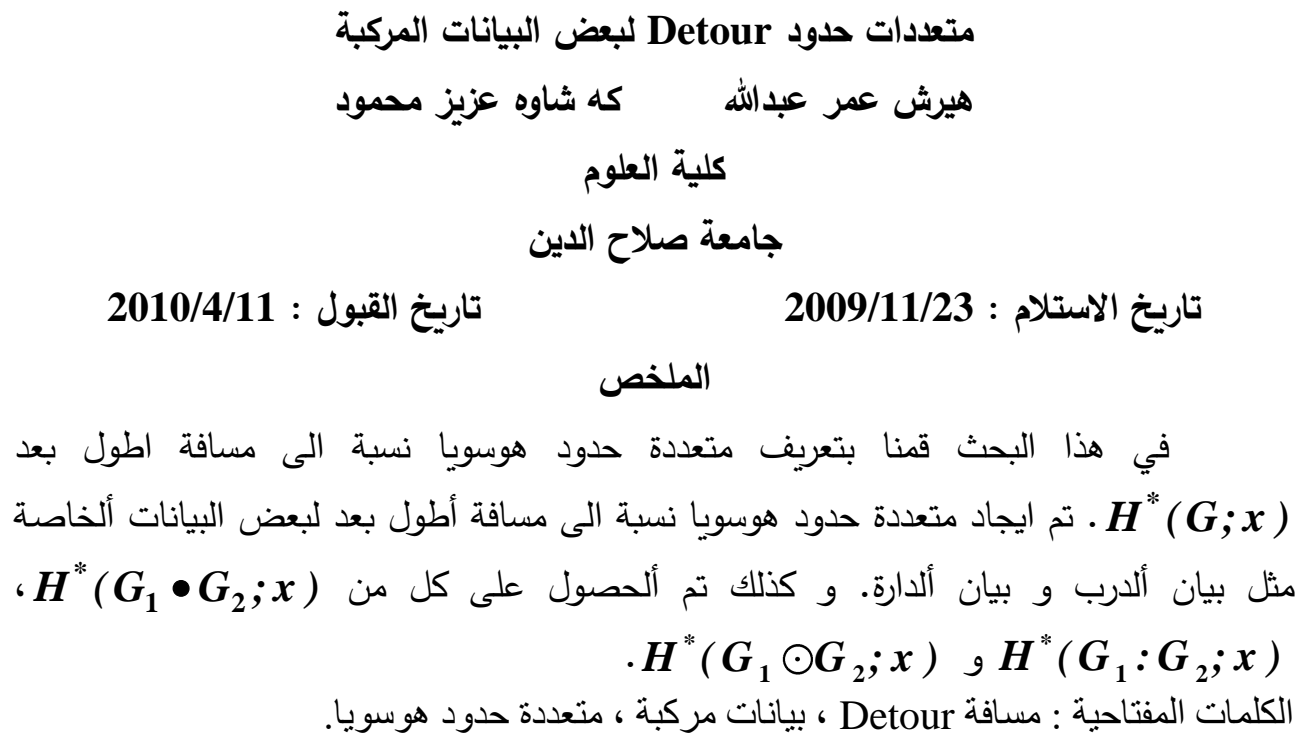

\section{Introduction}

The concept of Hosoya polynomial was first put forward in 1988 by Hosoya [1]. Several authors, such as [1], [2], [3], [4], [5], [6], [7], [8], [13] and [15] had obtained Hosoya polynomials for special graphs, graphs having some kind of regularity and for compound graphs obtained by using some well-known binary operations in graph theory. 
In this paper, we consider finite connected graphs without loops or multiple edges. For undefined concepts and notations see [9] and [12].

Ordinarily, when we wish to proceed from a point $\boldsymbol{A}$ to a point $\boldsymbol{B}$ we take a route which involves the least distance. We have all been faced with detour sign which require us to take a route from $\boldsymbol{A}$ to $\boldsymbol{B}$ that involves a greater distance. In any such detour route from $\boldsymbol{A}$ to $\boldsymbol{B}$ we assume that there is no possible shortcut along the route, for otherwise this should have been part of the route initially. When one is driving along such a detour, it sometimes seems that we are using the longest route possible from $\boldsymbol{A}$ to $\boldsymbol{B}$ (again subject to the "no shortcut" condition). In this paper we investigate longest detour routes in graphs.

The distance $\boldsymbol{d}(\boldsymbol{u}, \boldsymbol{v})$ between two vertices $\boldsymbol{u}$ and $\boldsymbol{v}$ in a connected graph $\boldsymbol{G}$ is the length of a shortest $\boldsymbol{u} \boldsymbol{v} \boldsymbol{v}$ path in $\boldsymbol{G}$. For a nonempty set $\boldsymbol{S}$ of vertices of $\boldsymbol{G}$, the subgraph $\langle\boldsymbol{S}\rangle$ of $\boldsymbol{G}$ induced by $\boldsymbol{S}$ as its vertex set while an edge of $\boldsymbol{G}$ belongs to $\langle\boldsymbol{S}\rangle$ if it joins two vertices of $\boldsymbol{S}$. If $\boldsymbol{P}$ is a $\boldsymbol{u}$-v path of length $\boldsymbol{d}(\boldsymbol{u}, \boldsymbol{v})$, then the subgraph $\langle\boldsymbol{V}(\boldsymbol{P})\rangle$ induced by the vertices of $\boldsymbol{P}$ is $\boldsymbol{P}$ itself. This observation suggests the following concept. The detour distance $\boldsymbol{d}^{*}(\boldsymbol{u}, \boldsymbol{v})$ between $\boldsymbol{u}$ and $\boldsymbol{v}$ in $\boldsymbol{G}$ is the length of a longest induced $\boldsymbol{u}-\boldsymbol{v}$ path, that is a longest $\boldsymbol{u}-\boldsymbol{v}$ path $\boldsymbol{P}$ for which $\langle\boldsymbol{V}(\boldsymbol{P})\rangle=\boldsymbol{P}$. An induced $\boldsymbol{u}-\boldsymbol{v}$ path of length $\boldsymbol{d}^{*}(\boldsymbol{u}, \boldsymbol{v})$ is called a detour path [10].

Observe that $\boldsymbol{d}^{*}(\boldsymbol{u}, \boldsymbol{v}) \geq \boldsymbol{d}(\boldsymbol{u}, \boldsymbol{v})$ for all vertices $\boldsymbol{u}$ and $\boldsymbol{v}$ of $\boldsymbol{G}$ and that $\boldsymbol{d}^{*}(\boldsymbol{u}, \boldsymbol{v})=\boldsymbol{d}(\boldsymbol{u}, \boldsymbol{v})=\mathbf{1}$ if and only if $\boldsymbol{u}$ and $\boldsymbol{v}$ are adjacent. Also, note that $d^{*}(u, v)=d^{*}(v, u)$ for all vertices $u$ and $v$ of $\boldsymbol{G}$. Therefore the detour distance is symmetric. However, the triangle inequality does not hold in general. Consider the wheel $\boldsymbol{W}_{\boldsymbol{p}}$ of order $\boldsymbol{p} \geq \mathbf{6}$ with center at the vertex $w$; then: $d^{*}(u, v)=p-3>2=d^{*}(u, w)+d^{*}(\boldsymbol{w}, v)$, for every two vertices $\boldsymbol{u}$ and $\boldsymbol{v}$ of $\boldsymbol{W}_{\boldsymbol{p}}, \boldsymbol{u}, \boldsymbol{v} \neq \boldsymbol{w}$, that are both adjacent to a common vertex $\boldsymbol{x} \neq \boldsymbol{w}$.

Therefore, in general, the detour distance is not a metric on the vertex set of $G[10]$.

The detour eccentricity $\boldsymbol{e}^{*}(\boldsymbol{v})$ of a vertex $\boldsymbol{v}$ is defined by $e^{*}(v)=\max \left\{d^{*}(v, w): w \in V(G)\right\}$. The detour eccentricity set $\boldsymbol{e}^{*}(\boldsymbol{G})$ of a connected graph $\boldsymbol{G}$ is the set consisting of all detour 
eccentricities of $\boldsymbol{G}$, that is $e^{*}(G)=\left\{e^{*}(v): v \in V(G)\right\}$. The detour radius $\boldsymbol{r a d}^{*}(G)$ of $\boldsymbol{G}$ is the minimum detour eccentricity, while the detour diameter diam* $(\boldsymbol{G})$ of $\boldsymbol{G}$ is the maximum detour eccentricity.

For completeness we define $\boldsymbol{d}^{*}(\boldsymbol{u}, \boldsymbol{v})=\mathbf{0}$ if and only $\boldsymbol{u}=\boldsymbol{v}$.

A connected graph $G$ is called a detour graph if $d^{*}(u, v)=d(u, v)$ for all vertices $\boldsymbol{u}$ and $\boldsymbol{v}$ of $\boldsymbol{G}$. No cycle of length $\mathbf{5}$ or more is a detour graph. On the other hand, all trees and all complete graphs are detour graphs. If $\boldsymbol{u}$ and $\boldsymbol{v}$ are distinct vertices of a graph $\boldsymbol{G}$ such that $\boldsymbol{d}^{*}(\boldsymbol{u}, \boldsymbol{v})=\mathbf{1}$ or $\mathbf{2}$, then $d^{*}(u, v)=d(u, v)[10]$, the converse is not true in general, that is if $d(u, v)=\mathbf{2}$, then $d^{*}(u, v) \geq \mathbf{2}$, as for the wheel $W_{p}, p \geq \mathbf{6}$.

The concept of Hosoya polynomial $\boldsymbol{H}(\boldsymbol{G} ; \boldsymbol{x})$ of a graph $\boldsymbol{G}$ was put forward by Hosoya[13], and defined as

$\boldsymbol{H}(\boldsymbol{G} ; \boldsymbol{x})=\sum_{k=0}^{\delta(G)} \boldsymbol{C}(\boldsymbol{G}, \boldsymbol{k}) \boldsymbol{x}^{k}$; where $\boldsymbol{C}(\boldsymbol{G}, \boldsymbol{k})$ is the number of pairs of vertices in $\boldsymbol{G}$ that are distance $\boldsymbol{k}$ apart, and $\boldsymbol{\delta}(\boldsymbol{G})$ is the diameter of the graph $\boldsymbol{G}$.

In this paper, the concept of Hosoya polynomials of detour distance of a connected graph $\boldsymbol{G}$ ( or simply detour Hosoya polynomial of a graph $\boldsymbol{G}$ ) has been defined by

$$
H^{*}(G ; x)=\sum_{k=0}^{\delta^{*}(G)} C^{*}(G, k) x^{k}=\sum_{\{u, v\} \subseteq V(G)} x^{d^{*}(u, v)}
$$

in which $\boldsymbol{C}^{*}(\boldsymbol{G}, \boldsymbol{k})$ is the number of pairs of vertices in $\boldsymbol{G}$ with detour distance $\boldsymbol{k}$, and $\boldsymbol{\delta}^{*}(\boldsymbol{G})$ is the detour diameter of $\boldsymbol{G}$. It is clear that if $\boldsymbol{G}$ is a detour graph, then $\boldsymbol{H}^{*}(\boldsymbol{G} ; \boldsymbol{x})=\boldsymbol{H}(\boldsymbol{G} ; \boldsymbol{x})$.

The sum $\boldsymbol{W}^{*}(\boldsymbol{G})$ of detour distances between all pairs of vertices of the graph $\boldsymbol{G}$ is known as the Wiener index of detour distance of the graph $\boldsymbol{G}$ (or simply detour Wiener index of the graph $\boldsymbol{G}$ ), that is

$$
W^{*}(G)=\sum_{u, v} d^{*}(u, v)
$$

where the sum is taken over all unordered pairs $\{\boldsymbol{u}, \boldsymbol{v}\}$ of distinct vertices in $\boldsymbol{G}$.

It is clear that 


$$
W^{*}(G)=\left.\frac{d}{d x} H^{*}(G ; x)\right|_{x=1} .
$$

We illustrate these ideas in the following example.

Example 1.1. Let $G$ be a graph of order $p=9$, depicted in figure 1.1(a).

It is clear that

$e^{*}\left(v_{1}\right)=5, e^{*}\left(v_{2}\right)=4, e^{*}\left(v_{3}\right)=4, e^{*}\left(v_{4}\right)=3, e^{*}\left(v_{5}\right)=4$, $e^{*}\left(v_{6}\right)=3, e^{*}\left(v_{7}\right)=4, e^{*}\left(v_{8}\right)=5$ and $e^{*}\left(v_{9}\right)=5$.

Hence

$$
e^{*}(G)=\{5,4,4,3,4,3,4,5,5\}, \text { diam }^{*}(G)=5 \text { and } \operatorname{rad}^{*}(G)=3 .
$$

A detour $v_{1}-v_{9}$ path is given in Figure 1.1(b). Therefore $d^{*}\left(v_{1}, v_{9}\right)=5$, and this gives us the maximum detour distance among all detour distances of pairs of vertices of $\boldsymbol{V}(\boldsymbol{G})$.

The path $\boldsymbol{P}^{\prime}$ is not a detour $\boldsymbol{v}_{\mathbf{1}}-\boldsymbol{v}_{9}$ path, because $\left\langle\boldsymbol{V}\left(\boldsymbol{P}^{\prime}\right)\right\rangle \neq \boldsymbol{P}^{\prime}$ (see figures 1.1(c) and 1.1(d)).

By direct calculations, we get that

$$
\begin{aligned}
& C^{*}(G, 0)=p=9, \quad C^{*}(G, 1)=10, \quad C^{*}(G, 2)=9, \\
& C^{*}(G, 3)=9, C^{*}(G, 4)=6 \text { and } C^{*}(G, 5)=2 .
\end{aligned}
$$

Hence, the detour Hosoya polynomial of $\boldsymbol{G}$ is

$$
H^{*}(G ; x)=9+10 x+9 x^{2}+9 x^{3}+6 x^{4}+2 x^{5}
$$

and

$$
W^{*}(G)=\left.\frac{d}{d x} H^{*}(G ; x)\right|_{x=1}=89
$$

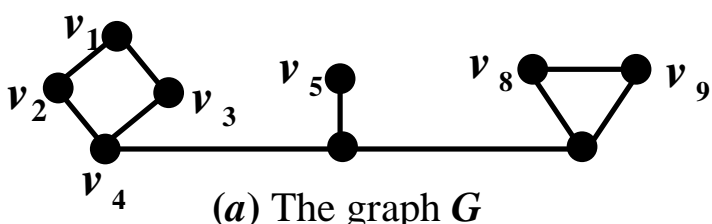

(a) The graph $\boldsymbol{G}$

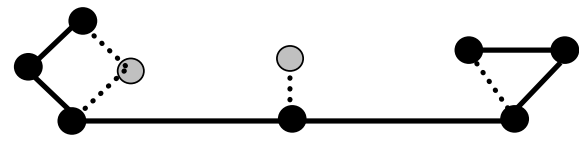

(c) The path $\boldsymbol{P}^{\prime}$

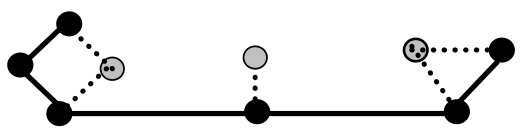

(b) The detour $v_{1}-v_{9}$ path

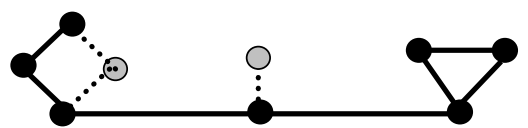

(d) $\left\langle V\left(P^{\prime}\right)\right\rangle$

Figure 1.1. 
In 1993, Gutman [8], established few additional properties of the respective graph polynomials. He obtained Hosoya polynomials of some special graphs and obtained formula for the Hosoya polynomials of some compound graphs, namely $\boldsymbol{G}_{\mathbf{1}} \bullet \boldsymbol{G}_{2}$ and $\boldsymbol{G}_{\mathbf{1}}: \boldsymbol{G}_{\mathbf{2}}$ which are defined in the following: Let $\boldsymbol{G}_{\mathbf{1}}$ and $\boldsymbol{G}_{\mathbf{2}}$ be vertex-disjoint connected graphs, and let $\boldsymbol{u} \in \boldsymbol{V}\left(\boldsymbol{G}_{1}\right)$ and $\boldsymbol{v} \in \boldsymbol{V}\left(\boldsymbol{G}_{2}\right)$. Then, the graph $\boldsymbol{G}_{\mathbf{1}} \bullet \boldsymbol{G}_{\mathbf{2}}$ is obtained from $\boldsymbol{G}_{\mathbf{1}}$ and $\boldsymbol{G}_{\mathbf{2}}$ by identifying the two vertices $\boldsymbol{u}$ and $\boldsymbol{v}$. This means that $\boldsymbol{G}_{\mathbf{1}}$ and $\boldsymbol{G}_{2}$ have exactly one vertex in common in the compound graph $\boldsymbol{G}_{\mathbf{1}} \bullet \boldsymbol{G}_{\mathbf{2}}$. The graph $\boldsymbol{G}_{\mathbf{1}}: \boldsymbol{G}_{\mathbf{2}}$ is obtained from $\boldsymbol{G}_{\mathbf{1}}$ and $\boldsymbol{G}_{\mathbf{2}}$ by introducing a new edge joining the two vertices $\boldsymbol{u}$ and $\boldsymbol{v}$. In this paper, formulas for $\boldsymbol{H}^{*}\left(\boldsymbol{G}_{\mathbf{1}} \bullet \boldsymbol{G}_{2} ; \boldsymbol{x}\right)$ and $\boldsymbol{H}^{*}\left(\boldsymbol{G}_{\mathbf{1}}: \boldsymbol{G}_{2} ; \boldsymbol{x}\right)$ in terms of the detour Hosoya polynomials of $\boldsymbol{G}_{\mathbf{1}}$ and $\boldsymbol{G}_{\mathbf{2}}$ will be obtained.

\section{Detour Hosoya Polynomials of Some Special Graphs}

Let $\boldsymbol{P}_{\boldsymbol{n}}, \boldsymbol{K}_{\boldsymbol{n}}$ and $\boldsymbol{S}_{\boldsymbol{n}}$ denotes the path, complete and star graphs of $\boldsymbol{n}$ vertices respectively. It is known that [10] all trees and complete graphs are detour graphs. This leads us to the following result.

\section{Proposition 2.1}

(a) $\quad H^{*}\left(P_{n} ; x\right)=\sum_{k=0}^{n-1}(n-k) x^{k}$

(b) $\quad H^{*}\left(K_{n} ; x\right)=n+\frac{1}{2} n(n-1) x$.

(c) $\quad H^{*}\left(S_{n} ; x\right)=n+(n-1) x+\left(\begin{array}{c}n-1 \\ 2\end{array}\right) x^{2}$

Proposition 2.2 Let $\boldsymbol{C}_{\boldsymbol{p}}$ be a cycle of order $\boldsymbol{p} \geq \mathbf{5}$, then

$$
H^{*}\left(C_{p} ; x\right)= \begin{cases}p\left(1+x+\sum_{k=\frac{p+1}{2}}^{p-2} x^{k}\right) & \text { if } p \text { is odd } \\ p\left(1+x+\frac{1}{2} x^{\frac{p}{2}}+\sum_{k=\frac{p}{2}+1}^{p-2} x^{k}\right) & \text { if } p \text { is even }\end{cases}
$$


$\underline{\text { Proof. }}$ Let $\boldsymbol{u}, \boldsymbol{v}$ be any two distinct vertices of $\boldsymbol{C}_{p}$. We will consider the following cases:

(1) If $u v \in E\left(C_{p}\right)$ then $d^{*}(u, v)=1$ and $C^{*}(G, 1)=p$.

(2) If $u v \notin E\left(C_{p}\right)$, then $d^{*}(u, v)=p-d(u, v)$,

where $\boldsymbol{d}(\boldsymbol{u}, \boldsymbol{v})$ denotes the ordinary distance.

We know that $[\mathbf{1 1}]$, for an odd $\boldsymbol{p}$, the ordinary Hosoya polynomial of $\boldsymbol{C}_{\boldsymbol{p}}$ is given by $H\left(C_{p} ; x\right)=p+p x+p \sum_{k=2}^{\frac{p-1}{2}} x^{k}$.

Hence

$$
H^{*}\left(C_{p} ; x\right)=p+p x+p \sum_{k=p-2}^{p-\frac{p-1}{2}} x^{k}
$$

or

$$
H^{*}\left(C_{p} ; x\right)=p+p x+p \sum_{k=\frac{p+1}{2}}^{p-2} x^{k} .
$$

Similarly, we prove the formula for the case when $\boldsymbol{p}$ is even.

This completes the proof.

Proposition 2.3 Let $\boldsymbol{W}_{p}$ be a wheel graph of $\boldsymbol{p} \geq \mathbf{6}$ vertices, then

$$
H^{*}\left(W_{p} ; x\right)=p+2(p-1) x+(p-1)\left\{\begin{array}{cc}
\sum_{k=\frac{p}{2}}^{p-3} x^{k}, & \text { if } p \text { is even } \\
\frac{1}{2} x^{\frac{p-1}{2}}+\sum_{k=\frac{p+1}{2}}^{p-3} x^{k}, & \text { if } p \text { is odd }
\end{array} .\right.
$$

Proof. For $u v \notin E\left(W_{p}\right), d_{W_{p}}^{*}(u, v)=d_{C_{p-1}}^{*}(u, v)$.

Hence, for $\boldsymbol{k} \geq \mathbf{2}$

$$
C^{*}\left(W_{P}, k\right)=C^{*}\left(C_{p-1}, k\right) .
$$

Thus,

$$
H^{*}\left(W_{p} ; x\right)=1+(p-1) x+H^{*}\left(C_{p-1}, x\right) .
$$

Now, using Proposition 2 we obtain the required result 
Proposition 2.4 Let $\boldsymbol{K}_{t, s}$ be a complete bipartite graph with partite subsets of sizes $t$ and $s$, then

$$
H^{*}\left(K_{t, s} ; x\right)=(t+s)+(t s) x+\left[\left(\begin{array}{l}
t \\
2
\end{array}\right)+\left(\begin{array}{l}
s \\
2
\end{array}\right)\right] x^{2} .
$$

Proof. Obvious

The following result gives us the Wiener index of the detour distance of the special graphs $\boldsymbol{P}_{n}, \boldsymbol{K}_{n}, \boldsymbol{S}_{n}, \boldsymbol{C}_{p}, \boldsymbol{W}_{p}$ and $\boldsymbol{K}_{t, \mathrm{~s}}$.

\section{Proposition 2.5}

(1) $W^{*}\left(P_{n}\right)=\frac{1}{6} n\left(n^{2}-1\right)$.

(2) $W^{*}\left(K_{n}\right)=\frac{1}{2} n(n-1)$.

(3) $W^{*}\left(S_{n}\right)=(n-1)^{2}$.

(4) For $p \geq 5, W^{*}\left(C_{p}\right)=\left\{\begin{array}{l}\frac{1}{8} p\left(3 p^{2}-12 p+17\right) \text {, if } p \text { is odd } \\ \frac{1}{8} p\left(3 p^{2}-12 p+16\right) \text {, if } p \text { is even }\end{array}\right.$.

(5) For $p \geq 6, W^{*}\left(W_{p}\right)=\left\{\begin{array}{l}\frac{1}{8}(p-1)\left(3 p^{2}-18 p+39\right), \text { if } p \text { is odd } \\ \frac{1}{8}(p-1)\left(3 p^{2}-18 p+40\right) \text {, if } p \text { is even }\end{array}\right.$.

(6) $W^{*}\left(K_{t, s}\right)=t s+t(t-1)+s(s-1)$.

\section{Detour Hosoya Polynomials of Some Compound Graphs}

Let $\boldsymbol{u}$ be a vertex of a connected graph $\boldsymbol{G}$ of order $\boldsymbol{p}$. The number of pairs of vertices of $\boldsymbol{G}$ containing the vertex $\boldsymbol{u}$ such that $\boldsymbol{d}_{G}^{*}(\boldsymbol{u}, \boldsymbol{v})=\boldsymbol{k}$, $\forall \boldsymbol{v} \in \boldsymbol{V}(\boldsymbol{G})$, will be denoted by $\boldsymbol{C}^{*}(\boldsymbol{u}, \boldsymbol{G} ; \boldsymbol{k})$.

We define the polynomial

$$
\boldsymbol{H}^{*}(\boldsymbol{u}, \boldsymbol{G} ; \boldsymbol{x})=\sum_{k=0}^{e^{*}(u)} \boldsymbol{C}^{*}(\boldsymbol{u}, \boldsymbol{G} ; \boldsymbol{k}) \boldsymbol{x}^{k}
$$

It is clear that

$$
H^{*}(G ; x)=\frac{1}{2} \sum_{u \in V(G)} H^{*}(u, G ; x)+\frac{1}{2} p
$$

Let $\boldsymbol{G}_{\mathbf{1}}$ and $\boldsymbol{G}_{\mathbf{2}}$ be two disjoint connected graphs of orders $\boldsymbol{p}_{\mathbf{1}}$ and $\boldsymbol{p}_{2}$ respectively. Moreover, let $\boldsymbol{w}$ be the vertex obtained by identifying the 
vertex $\boldsymbol{u}$ of $\boldsymbol{G}_{\mathbf{1}}$ with the vertex $\boldsymbol{v}$ of $\boldsymbol{G}_{2}$ in order to construct the compound graph $\boldsymbol{G}_{\mathbf{1}} \bullet \boldsymbol{G}_{\mathbf{2}}$. The compound graph $\boldsymbol{G}_{\mathbf{1}}: \boldsymbol{G}_{\mathbf{2}}$ is obtained by introducing a new edge joining the vertex $\boldsymbol{u}$ of $\boldsymbol{G}_{\mathbf{1}}$ with the vertex $\boldsymbol{v}$ of $\boldsymbol{G}_{\mathbf{2}}$.

Now, we are ready to present formulas for $\boldsymbol{H}^{*}\left(\boldsymbol{G}_{\mathbf{1}} \bullet \boldsymbol{G}_{2} ; \boldsymbol{x}\right)$ and $H^{*}\left(G_{1}: G_{2} ; \boldsymbol{x}\right)$ in terms of $\boldsymbol{H}^{*}\left(G_{1} ; \boldsymbol{x}\right)$ and $\boldsymbol{H}^{*}\left(\boldsymbol{G}_{2} ; \boldsymbol{x}\right)$.

$\underline{\text { Theorem }} 3.1$ If $\boldsymbol{G}_{1}$ and $\boldsymbol{G}_{2}$ are disjoint connected graphs, then

$$
\begin{aligned}
H^{*}\left(G_{1} \bullet G_{2} ; x\right) & =H^{*}\left(G_{1} ; x\right)+H^{*}\left(G_{2} ; x\right)+H^{*}\left(u, G_{1} ; x\right) . H^{*}\left(v, G_{2} ; x\right) \\
& -H^{*}\left(u, G_{1} ; x\right)-H^{*}\left(v, G_{2} ; x\right) .
\end{aligned}
$$

$\underline{\text { Proof: }}$ : Let $s, t$ be any two vertices of $G_{1} \bullet G_{2}$ such that $d_{G_{1} \bullet G_{2}}^{*}(s, t)=k$. We will consider the following cases:

(1) If $s, t \in V\left(G_{1}\right)$, then $C^{*}\left(G_{1} \bullet G_{2} ; k\right)=C^{*}\left(G_{1}, k\right)$, which produces the polynomial $H^{*}\left(\boldsymbol{G}_{\mathbf{1}} ; \boldsymbol{x}\right)$.

(2) If $s, t \in V\left(G_{2}\right)$, then $C^{*}\left(G_{1} \bullet G_{2} ; k\right)=C^{*}\left(G_{2}, k\right)$, which produces the polynomial $\boldsymbol{H}^{*}\left(\boldsymbol{G}_{\mathbf{2}} ; \boldsymbol{x}\right)$.

(3) $s \in V\left(G_{1}\right)$ and $t \in V\left(G_{2}\right)$ : In this case, any longest induced $(s, t)$ path $\boldsymbol{P}$ will contain the vertex $\boldsymbol{w}$. If $\boldsymbol{P}^{\prime}$ is a longest $(\boldsymbol{s}, \boldsymbol{w})$-path and $\boldsymbol{P}^{\prime \prime}$ is a longest $(\boldsymbol{t}, \boldsymbol{w})$-path with $\left\langle\boldsymbol{V}\left(\boldsymbol{P}^{\prime}\right)\right\rangle=\boldsymbol{P}^{\prime}$ and $\left\langle\boldsymbol{V}\left(\boldsymbol{P}^{\prime \prime}\right)\right\rangle=\boldsymbol{P}^{\prime \prime}$, then

$$
\boldsymbol{V}(\boldsymbol{P})=\boldsymbol{V}\left(\boldsymbol{P}^{\prime}\right) \cup \boldsymbol{V}\left(\boldsymbol{P}^{\prime \prime}\right), \text { and }\langle\boldsymbol{V}(\boldsymbol{P})\rangle=\left\langle\boldsymbol{V}\left(\boldsymbol{P}^{\prime}\right) \bigcup \boldsymbol{V}\left(\boldsymbol{P}^{\prime \prime}\right)\right\rangle,
$$

because no vertex of $\boldsymbol{P}^{\prime}$, other than $\boldsymbol{w}$ is adjacent with a vertex of $\boldsymbol{P}^{\prime \prime}$, other than $\boldsymbol{w}$.

Therefore $\boldsymbol{P}^{\prime} \bullet \boldsymbol{P}^{\prime \prime}=\langle\boldsymbol{V}(\boldsymbol{P})\rangle=\boldsymbol{P}$.

Hence, $d_{G_{1} \bullet G_{2}}^{*}(s, t)=d_{G_{1}}^{*}(s, w)+d_{G_{2}}^{*}(t, w)$.

This produces the polynomial $H^{*}\left(\boldsymbol{u}, \boldsymbol{G}_{1} ; \boldsymbol{x}\right) . \boldsymbol{H}^{*}\left(\boldsymbol{v}, \boldsymbol{G}_{2} ; \boldsymbol{x}\right)$. Notice that the polynomial $H^{*}\left(\boldsymbol{u}, \boldsymbol{G}_{\mathbf{1}} ; \boldsymbol{x}\right)$ is counted twice in the Cases (1) and (3), and also $H^{*}\left(v, G_{2} ; \boldsymbol{x}\right)$ is counted twice in the Cases (2) and (3).

Now, adding the polynomials obtained from the cases (1), (2) and (3), we get the required result.

Theorem 3.2 If $G_{1}$ and $G_{2}$ are disjoint connected graphs, then 
$H^{*}\left(G_{1}: G_{2}, x\right)=H^{*}\left(G_{1}, x\right)+H^{*}\left(G_{2}, x\right)+x . H^{*}\left(u, G_{1} ; x\right) . H^{*}\left(v, G_{2} ; x\right)$.

Proof. Let $\boldsymbol{s}, \boldsymbol{t}$ be any two distinct vertices of the compound graph $G_{1}: G_{2}$. We consider the following cases:

(1) If $s, t \in V\left(G_{1}\right)$, then we get the polynomial $H^{*}\left(G_{1} ; x\right)$.

(2) If $s, t \in V\left(G_{2}\right)$, then we get the polynomial $H^{*}\left(G_{2} ; x\right)$.

(3) $s \in V\left(G_{1}\right)$ and $t \in V\left(G_{2}\right)$ : In this case, any longest $(s, t)$-path will contains the edge $\boldsymbol{u v}$, and as in the proof of Theorem $\mathbf{6}$ (Case 3 ), this produces the polynomial

$$
\text { x. } H^{*}\left(u, G_{1} ; x\right) . H^{*}\left(v, G_{2} ; x\right)
$$

Now, adding the polynomials obtained from the cases (1), (2) and (3), we get the required result.

Definition 3.3 Let $\boldsymbol{G}_{1}$ and $\boldsymbol{G}_{\mathbf{2}}$ be disjoint connected graphs of orders $\boldsymbol{p}_{\mathbf{1}}$ and $\boldsymbol{p}_{\mathbf{2}}$, respectively. Let $\boldsymbol{G}_{\mathbf{2}}^{(\boldsymbol{i})}$ be the $\boldsymbol{i}^{\text {th }}$ copy of $\boldsymbol{G}_{\mathbf{2}}$. The Corona $\boldsymbol{G}_{\mathbf{1}} \odot \boldsymbol{G}_{\mathbf{2}}$, is the graph[13] constructed from $\boldsymbol{G}_{\mathbf{1}} \cup \boldsymbol{p}_{\mathbf{1}} \boldsymbol{G}_{\mathbf{2}}$ with additional edges $\bigcup_{i=1}^{p_{1}}\left\{v_{i} u: u \in V\left(G_{2}^{(i)}\right)\right\}$,

as depicted in Fig. 3.1, in which $V\left(G_{1}\right)=\left\{v_{1}, v_{2}, \ldots, v_{p_{1}}\right\}$.

It is clear that

$$
p\left(G_{1} \odot G_{2}\right)=p_{1}\left(1+p_{2}\right)=p
$$

and

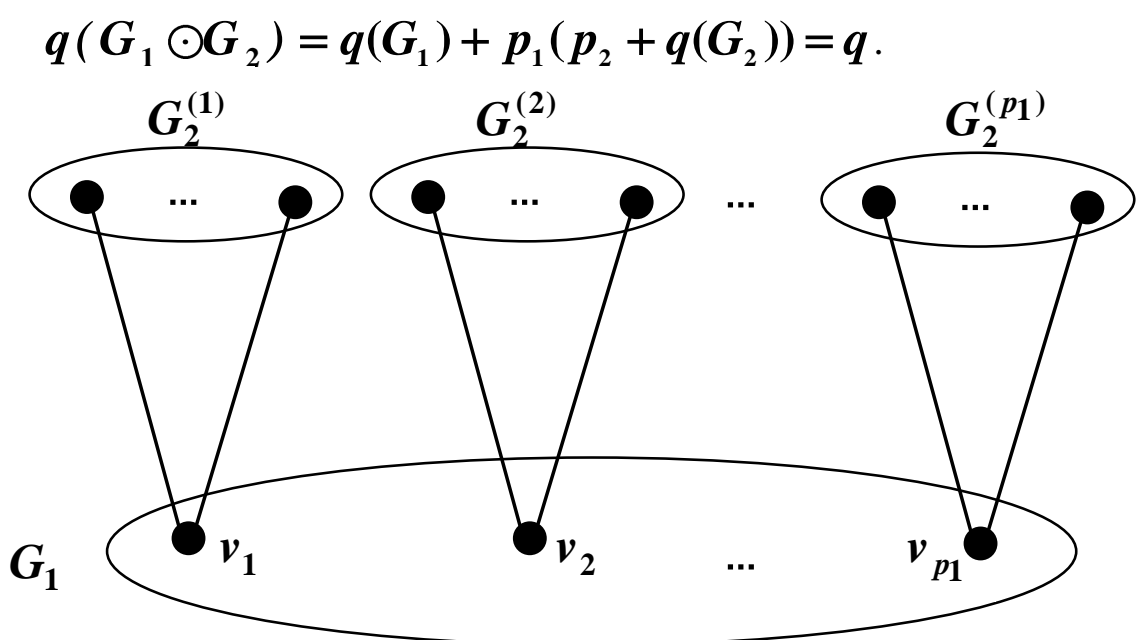

Fig. 3.1 The Corona $\boldsymbol{G}_{1} \odot \boldsymbol{G}_{2}$ 
The next theorem computes the detour Hosoya polynomial of the corona $\boldsymbol{G}_{\mathbf{1}} \odot \boldsymbol{G}_{\mathbf{2}}$.

Theorem 3.4 Let $\boldsymbol{G}_{\mathbf{1}}$ and $\boldsymbol{G}_{2}$ be two disjoint connected graphs, then

$$
\begin{aligned}
H^{*}\left(G_{1} \odot G_{2} ; x\right) & =\left(1+p_{2} x\right)^{2} H^{*}\left(G_{1} ; x\right)+p_{1} H^{*}\left(G_{2}, x\right) \\
& -p_{1} p_{2} x\left(1+p_{2} x\right) .
\end{aligned}
$$

$\underline{\text { Proof }}$. Let $\boldsymbol{s}, \boldsymbol{t}$ be any two distinct vertices of $\boldsymbol{G}_{\mathbf{1}} \odot \boldsymbol{G}_{\mathbf{2}}$. We will consider the following cases:

Case 1. If $s, t \in V\left(G_{1}\right)$, then we get the polynomial $H^{*}\left(G_{1} ; x\right)$.

Case 2. If $s, t \in V\left(G_{2}^{(i)}\right)$, for $i=1,2, \ldots, p_{1}$, then we get the polynomial $p_{1} H^{*}\left(G_{2} ; x\right)$.

Case 3. $s \in V_{2}^{(i)}$ and $t=v_{j}$ (or $s=v_{i}$ and $t \in V_{2}^{(j)}$ ) for $i, j=1,2, \ldots, p_{1}$, then

(i) If $\boldsymbol{i}=\boldsymbol{j}$, then we get the polynomial $\boldsymbol{p}_{1} \boldsymbol{p}_{2} \boldsymbol{x}$.

(ii) If $i \neq j$, then we get the polynomial $2 p_{2} x\left[H^{*}\left(G_{1} ; x\right)-p_{1}\right]$.

Case 4. If $s \in V_{2}^{(i)}$ and $t \in V_{2}^{(j)}$ for $i, j=1,2, \ldots, p_{1}, i \neq j$, then we get the polynomial $p_{2}^{2} x^{2}\left[H^{*}\left(G_{1} ; x\right)-p_{1}\right]$.

Now, adding the polynomials obtained from the above cases and simplifying, we get the required result. 


\section{REFERENCES}

[1] Abdullah, H. O. (2007). Hosoya polynomials of Steiner distance of Some graphs, Ph.D. Thesis, University of Salahaddin\Erbil, Erbil, Iraq.

[2] Ahmed, H. G. (2007). On Wiener Polynomials of n-Distance in Graphs, M.Sc. Thesis, Dohuk University, Dohuk, Iraq.

[3] Ali, A. A. and Sharaf, K. R. (1998). On Wiener polynomials of trees, Raf. J. Sc. Vol.9, No.1.

[4] Ali, A. M. (2005). Wiener polynomials of generalized distance in graphs, M. Sc. Thesis, Mosul University, Mosul, Iraq.

[5] Ali, A. A. and Ali, A. M. (2006). Wiener polynomials of the generalized distance for some special graphs, Rah. J. Com. Sc. And Maths., Vol.3, No.2, pp.103-120.

[6] Ali, A. A. and Saeed, W. A. (2006). Wiener polynomials of the strong product and semi-strong product, Raf. J. Sc. Vol.11, No.(3).

[7] Ali, A. A. and Saeed, W. A. (2006). Wiener polynomials of Steiner distance of graphs, J. of Applied Sciences, Vol.8, No.2.

[8] Ali, A. A. and Saeed, W. A. (2006). Wiener polynomials of the tensor product, Raf. J. Sc. Vol.17, No.1.

[9] Buckly, F. and Harary, F. (1990). Distance in Graphs, AddisonWesley, Redwood, California. U. S. A.

[10] Chartrand, G.; Johns, G. L. and Tian S. (1993). Detour distance in graphs, Annals of Discrete Mathematics, Vol. 55, pp. 127-136.

[11] Gutman, I. (1993). Some properties of the Wiener polynomial, Graph Theory Notes of New York, Vol.XXV, pp.13-18.

[12] Harary, F. (1969). Graph Theory, Addison-Wesley, Reading, Mass.

[13] Hosoya, H. (1988). On some counting polynomials in Chemistry, Discrete Applied Mathematics, Vol.19, pp.239-257.

[14] Saeed, W. (1999). Wiener polynomials of graphs, Ph.D. Thesis, University of Mosul.

[15] Sagan, B. E., Yeh, Y. N. and Zhang, P. (1996). The Wiener polynomial of a graph, International J. of Quantum Chemistry, Vol.60, pp.959-969. 